\title{
A SURVEY FOR RED VARIABLES IN THE MAGELLANIC CLOUDS
}

T. Lloyd Evans

South African Astronomical Observatory

ABSTRACT. One LMC and two SMC fields of $0.3 \mathrm{sq}$. deg. have been searched for red variables. Carbon stars of $\mathrm{V} \sim 16-17$ are common and are usually of small amplitude, while the LMC alone contains numerous faint $M$ type variables of small amplitude. M giants of small amplitude generally have much shorter periods than carbon stars. The LMC contains numerous Miras with a P-L relation similar to that of galactic Miras, while the SMC has few Miras but many bright red variables of large amplitude which have a steeper $\mathrm{P}-\mathrm{L}$ relation.

Lloyd Evans (1971a) made a study of Magellanic Cloud fields with the $1.9 \mathrm{~m}$ reflector from 1966-71. New and published results are discussed in the light of present ideas. The initial priority was to detect Mira variables and a sufficient number were found in a field in the Bar of the LMC to show that they obey a P-Mbol relation similar to that of galactic Miras (Glass \& Lloyd Evans 1981). Subsequent spectroscopic work shows that this relation holds for $M$, MS and carbon stars. Very few Miras have been found in the SMC; the distance is not so great as to be responsible. However the SMC contains much brighter red variables of large amplitude, including some of the Harvard variables as well as stars which are fainter in blue light because they are carbon stars (Lloyd Evans 1971b, 1980a, in preparation). The surface density of these stars is comparable to that of normal Miras in the LMC and the masses are estimated to be $\leq 7 \mathrm{M}_{\odot}$ (Lloyd Evans 1971b). Wood, Besse11 \& Fox (1981, preprint: WBF) deduce from extensive infrared photometry that all the large amplitude red variables are AGB stars with a range of mass and chemical composition which occupy a broad instability strip of slope in the P-Mbol diagram intermediate between the relations which would be fitted to LMC Miras and the more luminous variables separately. Removal of stars of small amplitude from this diagram shows two we11defined loci, linear in $\mathrm{M}_{\mathrm{bol}}-\log \mathrm{P}$, which represent the Mira and luminous Mira-like stars respectively and meet near $P=280$ days. Representatives of both Clouds fall on each locus and it remains to be seen whether a larger sample would fill the instability strip proposed by WBF. 
WBF and Froge1 (preprint) show that luminous red variables of small amplitude, all of which are $K$ or $M$ stars, are massive stars in the core $\mathrm{He}$ - or C - burning stage of evolution. These are present in moderate numbers in our fields but most small amplitude red variables have $\mathrm{V} \sim$ 16-17. Most of those in the SMC are carbon stars while there are also many $M$ stars in the LMC. The distribution of periods is quite different: carbon stars occupy a wide range from 170-360 days, with a few at longer or shorter periods (Lloyd Evans 1978), while nearly all M stars have $100 \leq \mathrm{P} \leq 150$ days.

\section{REFERENCES}

Glass, I.S. and Lloyd Evans, T., 1981. Nature, 291, pp 303-304. Lloyd Evans, T., 1971a. The Magellanic Clouds, pp 74-78, ed. Muller, A.B., Reide1, Dordrecht, Holland.

Lloyd Evans, T., 1971b. Observatory 91, pp 118-120.

Lloyd Evans, T., 1978. Mon. Not. R. astr. Soc., 183, pp 305-317.

Lloyd Evans, T., 1980. Mon. Not. R. astr. Soc., 193, pp 333-336.

Wood, P.R., Bessel1, M.F. and Fox, M.W., 1981. Proc. astr. Soc.

Australia, 4, pp 203-205. 